\title{
The Histogenesis of the Third Pathway of Colonic Carcinogenesis in Rats
}

\author{
CARLOS A. RUBIO \\ Gastrointestinal and Liver Research Laboratory, Department of Pathology, \\ Karolinska Institute and University Hospital, Stockholm, Sweden
}

\begin{abstract}
Background/Aim: Conventional (tubular or villous) adenomas, and the more recently described serrated adenomas, are non-invasive neoplasias that precede colon carcinomas in carcinogen-treated rats. In contrast, the histological steps antedating carcinomas in gut-associated lymphoid tissue $(G A L T)$ in rats, i.e. the third pathway of colonic carcinogenesis, remain unidentified. Aim of the study was to investigate the histological changes preceding colonic GALT carcinomas in Sprague-Dawley (SD) rats. Materials and Methods: Archived sections from previous experiments showing GALT mucosal domains in 292 rats were re-evaluated: 276 were injected with 1,2-dimethylhydrazine (DMH) suspended in ethylenediaminetetra-acetic acid (EDTA), and 16 were controls (8 EDTAtreated, and 8 untreated). Results: A total of 402 colonic GALT mucosal domains were found in the 292 rats: 382 in 276 DMHtreated, 10 in eight EDTA-treated, and 10 in eight-untreated rats. In DMH-treated rats, corrupted crypts (CCS; i.e. with asymmetric fission or abnormal crypt-alignment) were recorded in 50\% of the GALT domains (15\% had no dysplasia and $35 \%$ had epithelial dysplasia). Adenomas on top of GALT domains were found in 7\%, and GALT carcinomas in 53\%. Histology of the 146 colonic GALT carcinomas revealed highly differentiated carcinomas or signet-ring cell carcinomas. EDTA-treated and untreated animals showed no dysplastic CCS, or other neoplasia. Conclusion: This study demonstrated that GALT mucosal domains in carcinogen-treated rats often develop dysplastic CCS. Non-dysplastic CCS appear to act as scaffolds for the top-down replacement/transformation by dysplastic cells.
\end{abstract}

This article is freely accessible online.

Correspondence to: Carlos A. Rubio, MD, Ph.D., Gastrointestinal and Liver Pathology Research Laboratory, Department of Pathology, Karolinska Institute and University Hospital, 17176, Stockholm, Sweden. Tel: +46 851774527, Fax: +46 851774524, e-mail: Carlos.Rubio@ki.se

Key Words: Colon, rats, gut-associated lymphoid tissue, GALT, pathology, dysplasia, crypts, carcinoma.
Importantly, highly differentiated carcinomas were seen to evolve from dysplastic CCS and from adenomas, and signet-ring cell carcinomas from dysplastic goblet cells present at the base of crypts. This is the first study showing that non-invasive neoplastic lesions (dysplastic CCS and adenomas) antedate colonic GALT carcinomas in DMH-treated SD rats. The DMHSD paradigm permits detailed study of the histological events preceding GALT carcinoma under standard laboratory conditions.

Because of the high incidence of colorectal cancer (CRA) in humans, much research has focused on experimental animals as tools to monitor in detail the histological steps and biological attributes inherent to colon carcinogenesis. Fifty years ago, Druckey et al. reported colonic tumours in rats following the administration of 1,2-dimethylhydrazine (DMH) (1). Since then, more than 6,100 publications have appeared in PUBMED (2017-01-19, using the key words "colon cancer", and "rats". DMH and its carcinogenic metabolites [azoxymethane (AOM) and naethylazoxymethanol] have become the most commonly used compounds to study morphology, pathogenesis, prevention and treatment of experimentally induced colonic tumours in rodents (2-5).

Conventional (tubular or villous) adenomas (6), and the more recently described serrated adenomas (7), are non-invasive neoplasias that precede colon carcinomas in carcinogen-treated rats. Years ago, Deasy et al. (8) and Rubio et al. (9) reported that colonic carcinomas could also evolve in DMH-treated rats from mucosa built with lymphoid aggregates. In another experiment with DMH-treated rats, it was found that $37 \%$ of colonic neoplasias had a subjacent organized lymphoid nodule (10). These findings strongly suggest that carcinomas in rats evolve not only from conventional adenomas (6) and from serrated adenomas (7), but also from mucosa of gut-associated lymphoid tissue (GALT) $(8,9)$. However, the lesions which give rise to GALT carcinomas remain unidentified.

The purpose of the present investigation was to study in detail the histogenesis of GALT carcinomas in DMH-treated rats, particular attention being paid to the early stages prior to cancer development. 


\section{Materials and Methods}

Two hundred and seventy-six Sprague-Dawley (SD) male rats (Anticimex, Stockholm, Sweden) were injected s.c. with $21 \mathrm{mg} / \mathrm{kg}$ of body weight of DMH hydrochloride salt (MW, 133-02) (Kebo, Stockholm, Sweden) suspended in $1 \mathrm{ml} 1.5 \%$ ethylenediaminetetraacetic acid (EDTA) as a stabilizing agent, once a week. The treatment lasted 27 weeks. Eight additional SD rats received weekly injections containing $1 \mathrm{ml}$ EDTA solution for 27 weeks, and eight SD rats fed ad libitum for 27 weeks received no treatment. Thereafter the animals were sacrificed.

The specimens sampled at autopsy consisted of isolated tumours or of entire colons ('Swiss-roll' technique). The specimens were processed for histology and sections were stained with haematoxylin and eosin (H\&E).

Archived sections from previous experiments (10-14) were reviewed and those exhibiting colonic GALT mucosa, either normal or with neoplastic changes, were retrieved for this work.

Definitions. The normal colonic crypts of untreated rats are composed of test tube-shaped, close-packed crypts portraying straight axes, vertical to the muscularis mucosae.

Colonic GALT mucosal domain in SD rats: Colonic GALT domains are found in the anti-mesenteric border of the colon; they are typically organized into lymphoid aggregates within the mucosa and submucosa. Several authors described colonic GALT follicles to be covered by a mucosal lining having epithelial cuboidal cells, few or no goblet cells, $\mathrm{M}$ cells, and a subjacent primary lymphoid aggregate (without or with germinal centres) (15-17). In addition, colonic crypts with eosinophilic cytoplasm having few or no goblet cells were found here interspersed in the cuboidal epithelium in DMH-treated rats.

Corrupted colonic crypts (CCS) in GALT domains in DMHtreated rats: CCS in the GALT domain were regarded as those crypts showing asymmetric fission or aberrant crypt alignment (oblique or horizontal). Some were non-dysplastic and others exhibited epithelial dysplasia. Dysplastic CCS are lined by cells with hyperchromatic nuclei, cell stratification, eosinophilic cytoplasm with few or no goblet cells and occasionally, increased number of mitotic figures.

Adenomas on top of colonic GALT domains in DMH-treated rats: These are circumscribed neoplastic lesions composed of closepacked dysplastic CCS, found on top of colonic GALT domains (with or without carcinoma).

GALT carcinomas in DMH-treated rats: These are colonic GALT domains exhibiting neoplastic glands or signet ring cells invading into the submucosal layer or beyond. Three histological phenotypes were distinguished: highly differentiated carcinoma (HDC), signetring cell carcinomas (SRCC), and compound HDC/SRCC. Undifferentiated carcinoma was not found.

Statistical analysis. The non-parametric Mann-Whitney test was applied to compare difference between DMH-treated rats and controls. Statistical significance was defined as $p<0.05$.

The Ethical Committee of the Karolinska Institute, Stockholm, Sweden had approved the previous experiments (N 48/1989).

\section{Results}

Out of the 292 rats with colonic GALT mucosa, segmental biopsies were sampled in $90(31 \%)$ and Swiss roll specimens in the remaining $202(69 \%)$.
The sections reviewed were retrieved from the following groups: 276 from DMH-treated rats, eight from EDTAtreated rats and eight from untreated rats.

A total of 402 colonic GALT domains were detected: 382 in the $276 \mathrm{DMH}$-treated rats (1.38 GALT/rat), 10 in the eight EDTA-treated rats (1.25 GALT/rat), and 10 in the eight untreated rats (1.38 GALT/rat).

Histological findings in GALT domains in DMH-treated SD rats. Non-dysplastic CCS were recorded in 56 (15\%) out of the 382 colonic GALT domains, i.e. in $20 \%$ of the 276 rats (Figure 1). The epithelium in those crypts was usually eosinophilic, with few or no goblet cells.

Dysplastic CCs were present in 135 (35\%) out of the 382 colonic GALT domains, i.e. in $49 \%$ of the 276 DMH-treated rats (Figure 1). No dysplastic CCs were found in the colonic GALT domain of EDTA-treated rats nor of untreated rats. The difference in frequency between dysplastic CCs in $\mathrm{DMH}$-treated and in controls was significant $(p<0.05)$.

Out of the 135 GALT domains with dysplastic crypts, $24 \%(33 / 135)$ exhibited only one dysplastic CC and in the remaining $76 \%(102 / 135) \geq 2$ dysplastic CCs were recorded.

Adenomas on top of GALT domains were found in 20 (5\%) out of the 382 colonic GALT domains (7\% of the 276 DMH-treated rats).

GALT carcinomas were detected in $146(38 \%)$ out of the 382 colonic GALT domains (53\% of the $276 \mathrm{DMH}$-treated rats).

HDCs were found in $110(75 \%)$ at of the 146 colonic GALT carcinomas (Figure 2), SRCC in 29 (20\%) (Figure 2), mucinous carcinomas in four $(3 \%)$ and mixed carcinomas in the remaining three $(2 \%)$. The difference in frequency between HDC, and SRCCs/mixed carcinomas in GALT carcinomas was significant $(p<0.05)$.

HDCs were seen to evolve from dysplastic CCs (Figures 1 and 2) and from adenomas [16 HDCs (80\%) were found among the 20 adenomas], and SRCCs from dysplastic goblet cells present at the base of crypts (Figure 2).

In $23(16 \%)$ out of the 146 GALT carcinomas, the luminal surface was partially ulcerated.

Histological findings in GALT domains in control rats. No CCs with dysplasia, adenomas or carcinomas were found in the colonic GALT domain of EDTA-treated or of untreated rats.

\section{Discussion}

The colonic crypts replicate by symmetric fission, beginning at the base of the crypts and proceeding upwards until two identical, individual crypts are created (18-20). During crypt renewal, stem cells at the crypt bottom generate amplifying daughter cells that proliferate and differentiate while migrating upwards (18-20). APC normally down-regulates 

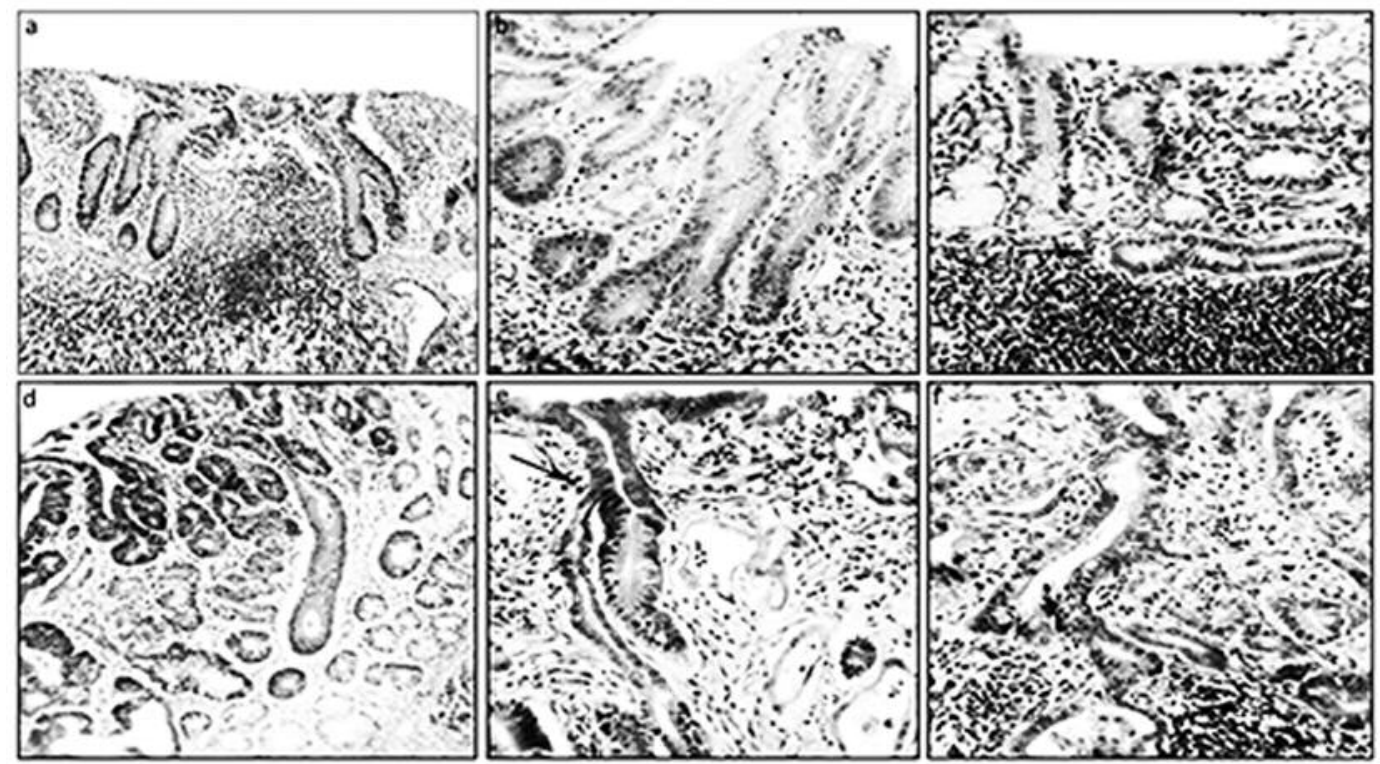

Figure 1. Histological features in colon tissue from dimethylhydrazine-treated Sprague-Dawley rats. Upper panel: Corrupted crypts with asymmetric fission $(a, b)$ or aberrant crypt alignment $(c$, arrow) in colonic gut-associated lymphoid tissue. Note few or no goblet cells, and absence of conventional dysplasia. Lower panel: Dysplastic corrupted crypts in colonic GALT-mucosa. Note highly differentiated carcinoma evolving from dysplastic corrupted crypts in e and f. Hematoxylin and eosin, $a, c, d: \times 10 ; b, e, f: \times 20$.

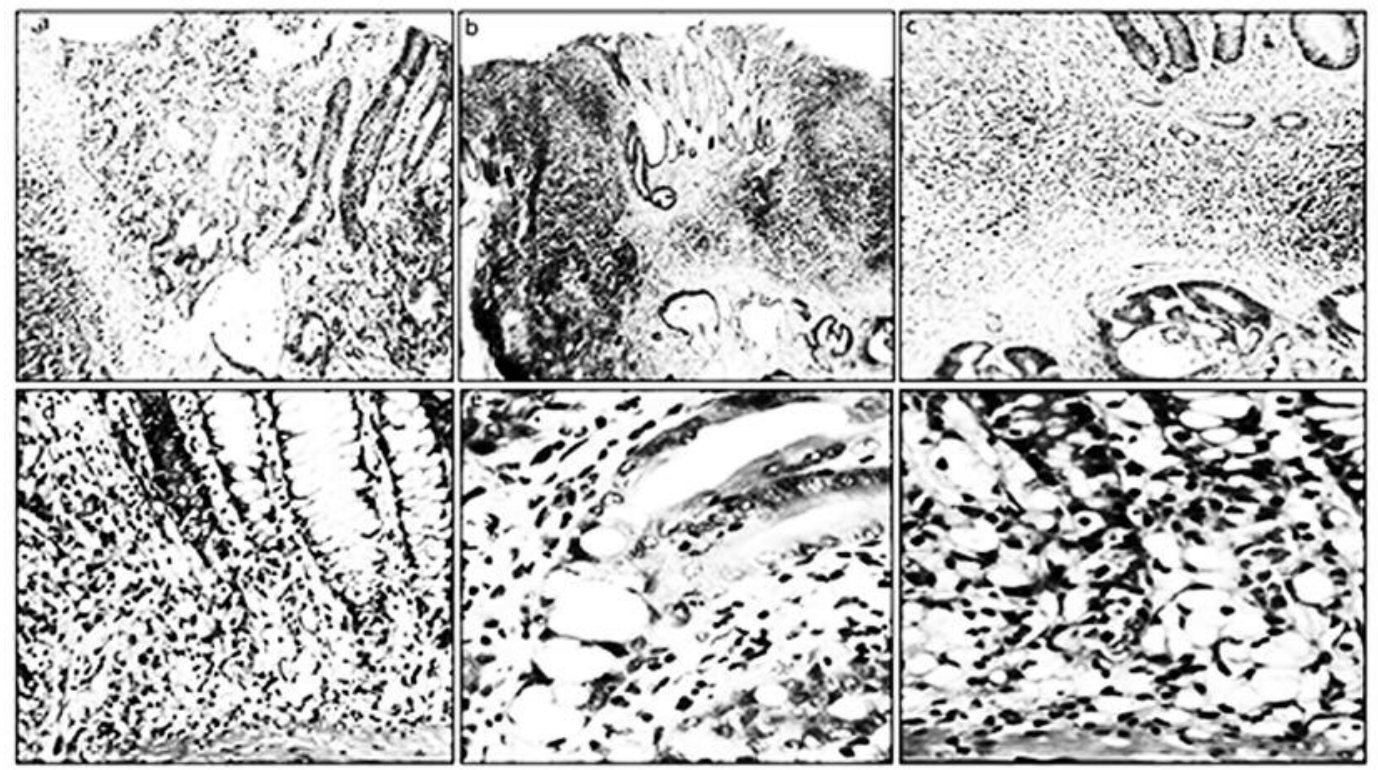

Figure 2. Histological features in colon tissue from in dimethylhydrazine-treated Sprague-Dawley rats. Upper panel: Highly differentiated carcinomas in colonic gut-associated lymphoid tissue (GALT) mucosa evolving from dysplastic corrupted crypts in a and b. Detail of highly differentiated carcinoma in c. Lower panel: Signet-ring cell carcinoma in colonic GALT mucosa (d), evolving from dysplastic goblet cells present at the base of crypts in e and f. Hematoxylin and eosin, $a: \times 10 ; b, d \times 4 ; c, f \times 20 ; e: \times 40$.

WNT signalling. The APC concentration is low at the crypt bottom and high at the top (the domain of differentiated cells). WNT signalling, in contrast, is high at the bottom (where stem cells reside) and low at the top. Hence, WNT and APC gradients are important in crypt formation and regulation. Since both APC and WNT signalling components (e.g. survivin) are required for mitosis, this mechanism establishes a zone in the lower crypt where conditions are 
optimal for maximal cell division and crypt orientation. Mutation of the $A P C$ gene causes abnormal crypt fission, disorientation of the crypts, and increased crypt production, leading to colorectal adenoma (20). Thus, the asymmetric fission of non-dysplastic crypts in GALT domains found here (i.e. CC), might be the result of mutations in the APC gene triggered by the carcinogen DMH.

In DMH-treated rats, dysplastic CCS were found in $50 \%$ of the colonic GALT domains, i.e. in nearly $70 \%$ of the DMH-treated rats. Colonic GALT carcinomas developed in more than $50 \%$ of the DMH-treated rats. HDCs were seen to evolve from dysplastic CCs and from adenomas (with dysplastic CCs), and SRCC from dysplastic goblet cells present at the base of crypts.

In conclusion, this study demonstrated that GALT domains of carcinogen-treated rats often develop dysplastic CCs. Nondysplastic CCs appeared to act as scaffolds for the top-down replacement/transformation by dysplastic cells. Importantly, HDCs were seen to evolve from CCs with dysplasia and from adenomas, and SRCC from dysplastic goblet cells present at the base of crypts. This is the first study showing that noninvasive neoplastic lesions often antedate colonic GALT carcinomas in carcinogen-treated SD rats.

The DMH-SD paradigm permits the detailed study of histological events preceding GALT carcinoma, i.e. the third pathway of colonic carcinogenesis (21) under standard laboratory conditions.

\section{Author Contributions}

The Author reviewed archived sections from previous experiments, compiled the data and wrote the article.

\section{Conflicts of Interest}

None.

\section{References}

1 Druckrey H, Preussmann R, Matzkies F and Ivankovic S: Selective production of intestinal cancer in rats by 1,2dimethylhydrazine. Naturwissenschaften 54: 285-286, 1967.

2 Rubio CA, Wallin B, Ware J, Sveander M and Duvander A: Effect of indomethacin in autotransplanted colonic tumors. Dis Colon Rectum 32: 488-491, 1989.

3 Rubio C A and Rivera F: Quantification of acid mucins in the descending colon of rats having simultaneously growing colonic tumors. APMIS 99: 993-996, 1991.

4 Rubio C A: Apoptotic differences in experimentally induced colorectal rat tumours. Apoptosis 3: 35-39, 1998.

5 Rubio C A, Wallin B, Sveander M and Duvander A: A model for the study of metastases from colonic tumors by autotransplantation. Dis Colon Rectum 30: 884-887, 1987.
6 Boivin GP, Washington K, Yang K, Ward JM, Pretlow TP, Russell R, Besselsen DG, Godfrey VL, Doetschman T, Dove WF, Pitot $\mathrm{HC}$, Halberg RB, Itzkowitz SH, Groden $\mathrm{J}$ and Coffey RJ: Pathology of mouse models of intestinal cancer: consensus report and recommendations. Gastroenterology 124: 762-77, 2003.

7 Rubio CA: Traditional serrated adenomas and serrated carcinomas in carcinogen-treated rats. J Clin Pathol pii: jclinpath-2016-204037. doi: 10.1136, 2016.

8 Deasy JM, Steele G Jr., Ross DS, Lahey SJ, Wilson RE and Madara J: Gut-associated lymphoid tissue and dimethylhydrazineinduced colorectal carcinoma in the Wistar/Furth rat. J Surg Oncol 24: 36-40, 1983.

9 Rubio CA: Lymphoid tissue-associated colonic adenocarcinomas in rats. In Vivo 1: 61-64, 1987.

10 Rubio CA, Shetye J and Jaramillo E: Non-polypoid adenomas of the colon are associated with subjacent lymphoid nodules. An experimental study in rats. Scand J Gastroenterol 34: 504-508, 1999.

11 Rubio CA, Nylander G, Sveander M, Duvander A and Alun ML: Minimal invasive carcinoma of the colon in rats. Am J Pathol 123: 161-165, 1986.

12 Rubio CA, Nylander G, Wahlin B, Sveander M, Duvander A and Alun ML: Monitoring the histogenesis of colonic tumors in the Sprague-Dawley rat. J Surg Oncol 3: 225-228, 1986.

13 Rubio CA, Jaramillo E and Sethye J: Adenomas and carcinomas may be histologically detected in apparently normal colonic mucosa. A study of carcinogen-treated rats. In Vivo 15: 299-301, 2001.

14 Rubio CA and Takayama S: Difference in histology and size in colonic tumors of rats receiving two different carcinogens. J Environ Pathol Toxicol Oncol 13: 191-197, 1994.

15 Neutra M, Mantis N and Kraehenbuhl J P: Collaboration of epithelial cells with organized mucosal lymphoid tissues. Nature Immunol 2: 1004-1009, 2001.

16 Butler, JE and Sinkora M: The enigma of the lower gut-associated lymphoid tissue (GALT). J Leukoc Biol 94: 259-270, 2013.

17 Autenrieth IB and Firsching R: Penetration of M cells and destruction of Peyer's patches by Yersinia enterocolitica: an ultrastructural and histological study. J Med Microbiol 44: 285294, 1996.

18 St Clair WH and Osborne JW: Crypt fission and crypt number in the small and large bowel of postnatal rats. Cell Tissue Kinet 18: 255-262, 1985

19 Rubio CA: Putative stem cells in mucosas of the esophagogastrointestinal tract. Chapter 10. In: Stem Cell, Regenerative Medicine and Cancer. Singh SR (ed.). Haupauge, NY, USA: Nova Science Publishers, Inc., pp. 279-308. 2011.

20 Boman BM and Fields JZ: An APC/WNT counter-current-like mechanism regulates cell division along the human colonic crypt axis: a mechanism that explains how apc mutations induce proliferative abnormalities that drive colon cancer development. Front Oncol 3: 244-252, 2013.

21 Rubio CA: Three pathways of colonic carcinogenesis in rats. Anticancer Res 37: 15-20, 2017.

Received January 22, 2017

Revised February 24, 2017

Accepted February 28, 2017 